\title{
Enhancing IgG distribution to lung mucosal tissue improves protective effect of anti-Pseudomonas aeruginosa antibodies
}

\author{
M. Jack Borrok, ${ }^{1}$ Antonio DiGiandomenico, ${ }^{2}$ Nurten Beyaz, ${ }^{1}$ Gabriela M. Marchetti, ${ }^{1,3}$ \\ Arnita S. Barnes, ${ }^{1}$ Kristen J. Lekstrom, ${ }^{1}$ Sandrina S. Phipps, ${ }^{1}$ Michael P. McCarthy, ${ }^{2}$ Herren Wu, ${ }^{1}$ \\ William F. Dall'Acqua, ${ }^{1}$ Ping Tsui, ${ }^{1}$ and Ruchi Gupta ${ }^{3}$ \\ ${ }^{1}$ Antibody Discovery and Protein Engineering, ${ }^{2}$ Microbial Sciences, and ${ }^{3}$ Cardiovascular, Renal, and Metabolic Disease, \\ Medlmmune, Gaithersburg, Maryland, USA.
}

\begin{abstract}
IgC antibodies are abundantly present in the vasculature but to a much lesser extent in mucosal tissues. This contrasts with antibodies of the IgA and IgM isotype that are present at high concentration in mucosal secretions due to active delivery by the polymeric lg receptor (plgR). IgC is the preferred isotype for therapeutic mAb development due to its long serum half-life and robust Fc-mediated effector function, and it is utilized to treat a diverse array of diseases with antigen targets located in the vasculature, serosa, and mucosa. As therapeutic IgC antibodies targeting the luminal side of mucosal tissue lack an active transport delivery mechanism, we sought to generate IgC antibodies that could be transported via plgR, similarly to dimeric IgA and pentameric IgM. We show that an anti-Pseudomonas aeruginosa IgC fused with plgR-binding peptides gained the ability to transcytose and be secreted via plgR. Consistent with these results, plgR-binding IgC antibodies exhibit enhanced localization to the bronchoalveolar space when compared with the parental IgC antibody. Furthermore, plgR-binding mAbs maintained Fc-mediated functional activity and promoted enhanced survival compared with the parental mAb in a $P$. aeruginosa acute pneumonia model. Our results suggest that increasing IgC accumulation at mucosal surfaces by plgR-mediated active transport can improve the efficacy of therapeutic mAbs that act at these sites.
\end{abstract}

Conflict of interest: All authors are employees of, or have received funding from, Medlmmune, a member of the AstraZeneca group.

Submitted: October 5, 2017

Accepted: May 8, 2018

Published: June 21, 2018

\section{Reference information:}

JCI Insight. 2018;3(12):e97844.

https://doi.org/10.1172/jci.

insight. 97844.

\section{Introduction}

Immunoglobulin isotype classes ( $\mathrm{IgG}, \operatorname{IgA}, \operatorname{IgM}, \operatorname{IgD}$, and $\operatorname{IgE}$ ) have distinct roles in humoral immunity and are distributed differently throughout the body. Of these, the IgG isotype is overwhelmingly favored for therapeutic $\mathrm{mAbs}$ for numerous reasons. Primarily, the neonatal $\mathrm{Fc}$ receptor ( $\mathrm{FcRn}$ ) confers long serum half-life, and $\mathrm{Fc} \gamma$ receptors ( $\mathrm{F} c \gamma \mathrm{Rs}$ ) impart immune effector function to IgG antibodies exclusively. Additionally, the production, purification, and overall manufacturability properties of $\mathrm{IgG} \mathrm{mAbs}$ are considered superior to other isotypes, which generally exhibit more complex oligomerization and glycosylation patterns.

The clinical preference for the IgG isotype limits therapeutic antibodies to certain types of (Fc $\gamma \mathrm{R}$-mediated) immune engagement and governs distribution throughout the body. IgG cannot be secreted to mucosal tissues like IgA and IgM, and it must rely on passive transport or transudation to accumulate at these sites. Many IgG antibodies approved or under clinical evaluation treat diseases affecting mucosal organs. These include antibodies targeting pulmonary diseases caused by viruses or bacteria (1-3), as well as other respiratory (4) and intestinal diseases (5). For therapeutic antibodies targeting antigens located in these tissues, the mainly vascular distribution of IgG could be unfavorable compared with IgA and IgM, which can be transported into the mucosal lumen at high levels. Indeed, systemically administered IgG levels in bronchoalveolar lavage (BAL) fluid have been reported to be approximately 1,000 times lower than levels of IgG in serum (6-8).

The polymeric Ig receptor (pIgR) facilitates the conversion of dimeric $\operatorname{IgA}(\operatorname{dg} \mathrm{A})$ and pentameric IgM to their secreted forms (sIgA and $\operatorname{sgM}$ ), while transporting these molecules from the lamina propria of mucosal tissues to the lumen $(9,10)$. The secretory forms of IgA and IgM differ from those found in serum in that the extracellular portion of pIgR (after facilitating luminal transport) is proteolytically 
cleaved and remains bound to these Ig as the secretory component (11). The secretory component confers stability and protease resistance to IgA in the mucosal environment and enhances the protective ability of these molecules (12-14).

In this study, we sought to determine whether the distribution and efficacy of an IgG antibody could be altered by endowing it with the ability to bind and be transported by pIgR. We chose Cam-003, a human $\mathrm{IgG} \mathrm{mAb}$ that targets the polysaccharide synthesis locus (Psl) exopolysaccharide of Pseudomonas aeruginosa (P. aeruginosa) (15), as our model therapeutic antibody. $P$. aeruginosa is an important respiratory pathogen and a significant cause of acute pneumonia in ventilated subjects. In addition, $P$. aeruginosa is also a major cause of chronic lung infections in cystic fibrosis patients and leads to persistent lung function damage, ultimately causing death in this patient population. Psl is a serotype-independent exopolysaccharide produced by $P$. aeruginosa that mediates attachment to epithelial cells and is important in the formation and maintenance of antibiotic recalcitrant biofilms $(16,17)$. In addition, Psl prevents complement deposition on the bacterial surface, thus allowing $P$. aeruginosa to escape immune recognition by host effector cells (18, 19). Cam-003 targeting of Psl mediates potent complement-dependent opsonophagocytic killing (OPK) of $P$. aeruginosa by host effector cells and confers significant protective activity against diverse $P$. aeruginosa strains in a murine lung infection model (15).

In this work, we show that Cam-003 fused to pIgR-binding peptides binds to pIgR and undergoes pIgR-mediated transcytosis. In addition, i.v. administration of the $\mathrm{mAb}$ in mice resulted in greater accumulation of pIgR peptide-fused antibodies in BAL fluid when compared with the parental IgG $\mathrm{mAb}$. Consistent with these results, i.v. administration of $\mathrm{pIgR}$ peptide-fused Cam-003 to mice 24 hours before infection resulted in enhance survival against lethal $P$. aeruginosa lung infection compared with animals receiving the parental Cam-003 mAb. Our results demonstrate that conferring therapeutic IgG antibodies with pIgR binding capabilities could enhance the accumulation of therapeutic IgG antibodies targeting bacteria to the lung lumen.

\section{Results}

Generation and characterization of pIgR-binding peptide antibody fusions. Peptides have previously been identified that bind to $\mathrm{pIgR}$ and promote transcytosis of phage particles or other cargo $(20,21)$. To assess whether addition of these peptides to IgGs can alter antibody biodistribution, several of these peptides were generated as C-terminal fusions to the heavy chain of Cam-003 (a human IgG1 antibody that binds to the Psl exopolysaccharide of P. aeruginosa) (15). A GGGGS linker was employed to link the C-terminus of the antibody with the peptides tested. Several peptides known to bind $\operatorname{PIgR}(20,21)$, as well as full-length human and mouse J-chains (known to facilitate pIgR binding in IgA and IgM), were constructed. Many of the antibody-peptide fusions (including the J-chain fusions) had extremely poor expression or severe aggregation issues (Supplemental Table 1; supplemental material available online with this article; https://doi. org/10.1172/jci.insight.97844DS1). However, 2 peptides with the sequences QRNPRLRLIRRHPTLRIPPI and SAMFVPFDIAVGVRDGQQGLGGSRRKGARLREAISSYAE (referred to hereafter as QRN and SAM) were well expressed and had high monomeric content. Binding to either human (h) or murine (m) pIgR was then evaluated via ELISA (Figure 1). Cam-003-QRN was found to bind to m-pIgR with a half maximal effective concentration $\left(\mathrm{EC}_{50}\right.$ ) of $0.50 \mu \mathrm{g} / \mathrm{ml}$ and to h-pIgR with an $\mathrm{EC}_{50}$ of $0.52 \mu \mathrm{g} / \mathrm{ml}$. Cam003-QRN binding exceeded that of human $\mathrm{dIgA}$, a natural ligand for $\mathrm{pIgR}$, which bound with an $\mathrm{EC}_{50}$ of $11.7 \mu \mathrm{g} / \mathrm{ml}$ for h-pIgR and $8.7 \mu \mathrm{g} / \mathrm{ml}$ for m-pIgR. Cam-003-SAM had only minimal binding to pIgR via ELISA and was not carried forward for further experiments.

Generation of the Cam-003-QRN variants. Cam-003-QRN bound effectively to both human and murine pIgR, yet mass spectrometric analysis of purified Cam-003-QRN revealed that protease cleavage occurred at 2 regions within the QRN peptide sequence (Supplemental Figure 1). The major species (species 1) observed was full length or missing the C-terminal isoleucine or the C-terminal dipeptide PI. An additional less abundant species observed was cleaved in the RLRL region midpeptide (species 2). Peak heights were used to estimate the abundance of the 2 species (Supplemental Figure 1). Analysis of 3 separate batches revealed QRN species 1 was $\sim 70 \%$ abundant upon purification, whereas $\mathrm{QRN}$ species 2 was present at $\sim 30 \%$ abundance. To increase the amount of species 1 QRN peptide, several mutants were generated in the RLRL sequence of the QRN peptide fusion (Supplemental Table 2). Of these, 1 mutant with lysines replacing 2 arginine residues in this sequence (referred to as KLKL) could be expressed consistently with high species 1 content ( $~ 85 \%)$ and maintained binding to h-pIgR and m-pIgR similarly to the parental QRN fusion (Figure 1). Cam-003-KLKL bound 

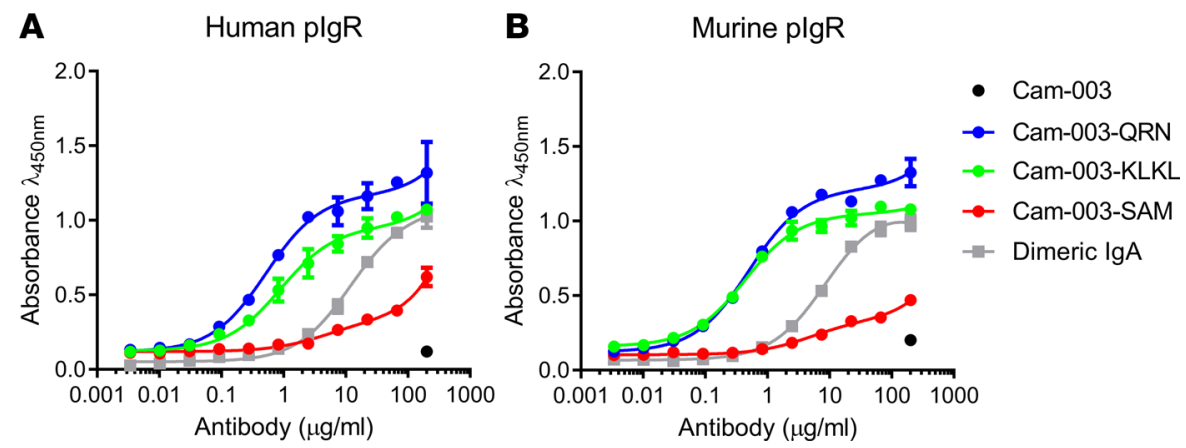

Figure 1. Cam-003 peptide fusions bind to human and murine plgR. Binding of C-terminal peptide antibody fusions to plgR was determined by ELISA. Cam-003 alone showed no binding to either human (A) or murine (B) plgR at the highest concentration tested $(200 \mu \mathrm{g} / \mathrm{ml})$. Cam-003-QRN and its derivative Cam-003-KLKL had improved binding to plgR compared with the natural plgR ligand dimeric IgA, whereas another peptide fusion Cam-003-SAM exhibited poorer binding $(n=4)$. Data are representative of at least 2 independent experiments.

to m-pIgR with an $\mathrm{EC}_{50}$ of $0.43 \mu \mathrm{g} / \mathrm{ml}$ and to $\mathrm{h}-\mathrm{pIgR}$ with an $\mathrm{EC}_{50}$ of $0.89 \mu \mathrm{g} / \mathrm{ml}$ via ELISA. Other mutations within the RLRL sequence of the QRN peptide either did not retain pIgR binding or had less full-length (species 1) heavy chains observed than the original QRN peptide.

IgG peptide fusions transcytosis in pIgR-expressing cells. As the QRN C-terminal antibody fusion and engineered KLKL variant bound to the pIgR receptor, we next sought to determine whether these antibody fusions could be cargo for pIgR-mediated transcytosis. Stable human and murine pIgR expressing Madin-Darby canine kidney (MDCK) cells were generated to assess pIgR-mediated transcytosis (22). Cam003 and Cam-003 peptide fusions were then evaluated in transwell assays. Antibodies were added in the bottom (basolateral side) chamber, and transcytosis was measured by quantifying antibody in the top (apical) chamber (Figure 2A). In either h-pIgR-or m-pIgR-expressing MDCK cells, Cam-003 levels in the apical chamber were at low levels, similar to untransfected MDCK cells (Figure 2B). After 24 hours, increased transcytosis was observed for both Cam-003-KLKL and Cam-003-QRN when compared with Cam-003 alone in wells with both h-pIgR- and m-pIgR-expressing MDCK cells (Figure 2B).

pIgR-binding IgG fusion levels increased in BAL. We then determined how Cam-003-QRN and Cam-003KLKL fusions would alter antibody localization to the lung lumen when administered i.v. to mice. Cam003-QRN, Cam-003-KLKL, and Cam-003 IgG were dosed via tail-vein injection into mice, followed by collection of BAL fluid 48 hours after administration. Serum samples taken at 24 and 48 hours were also collected, and antibody levels were determined by biolayer interferometry (OCTET). Serum levels were similar between Cam-003 and the Cam-003 pIgR peptide fusions (Figure 3A). However, Cam-003-QRN and Cam-003-KLKL exhibited 2.7- and 3.9-fold higher IgG concentrations, respectively, in BAL fluid 48 hours after antibody administration when compared with Cam-003 (Figure 3B).

Assessing antibody fusions for OPK activity against $P$. aeruginosa. Prior to testing the pIgR-binding Cam003 fusions in a $P$. aeruginosa infection model, we first assessed whether these antibodies maintain the ability to promote $\mathrm{Fc}$-mediated phagocyte-dependent killing of $P$. aeruginosa. It has previously been shown that Cam-003 promotes OPK of $P$. aeruginosa and that this mechanism of action correlates with protective activity in vivo (15). Robust complement-dependent OPK of the $P$. aeruginosa strain PAO1 was observed with Cam-003 with $\mathrm{EC}_{50}$ values of $0.11 \mu \mathrm{g} / \mathrm{ml}$ (Figure 4A). Cam-003-QRN- and Cam-003-KLKL-mediated OPK was similar to Cam-003 alone ( $\mathrm{EC}_{50}$ values 0.09 and $0.15 \mu \mathrm{g} / \mathrm{ml}$, respectively), suggesting the C-terminal peptide fusions did not significantly impair OPK activity. Samples lacking antibody (media only) or with an isotype control IgG failed to elicit OPK activity against $P$. aeruginosa (Figure 4A). For this experiment, human serum (depleted of Pseudomonas-binding antibodies) was used as a complement source. OPK with an additional commonly used complement source (baby rabbit complement) was also evaluated (Supplemental Figure 3). Again, Cam-003, Cam-003-QRN, and Cam-003-KLKL displayed robust OPK activity, with the peptide fusions having slightly lower $\mathrm{EC}_{50}$ values than Cam-003.

pIgR-binding peptide fusion mAbs exhibit enhanced protective activity in vivo. Given that Cam-003 pIgR fusion mAbs could still elicit OPK activity, we next sought to evaluate whether they provided improved protection in vivo in comparison with the parental Cam- $003 \mathrm{mAb}$ and a control IgG. The pIgR-binding peptide fusions, 
A

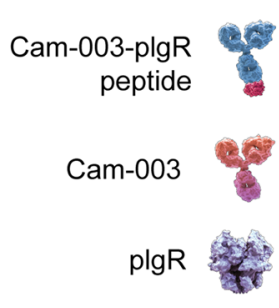

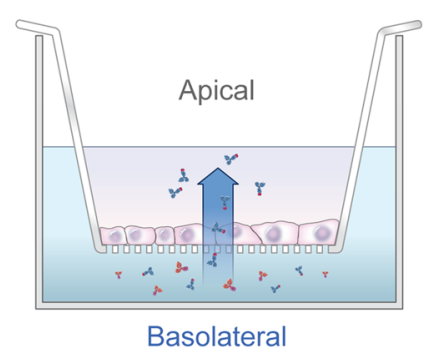

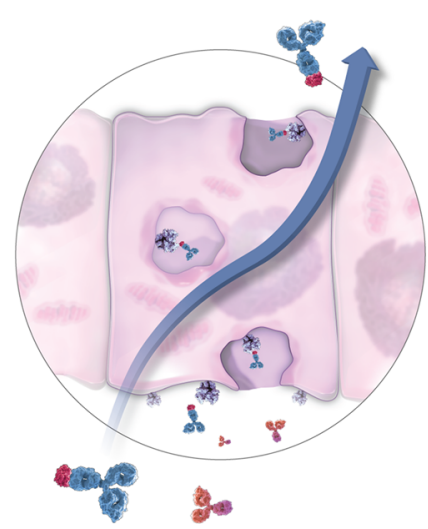

B

\author{
Transcytosis of plgR Binding Antibodies
}

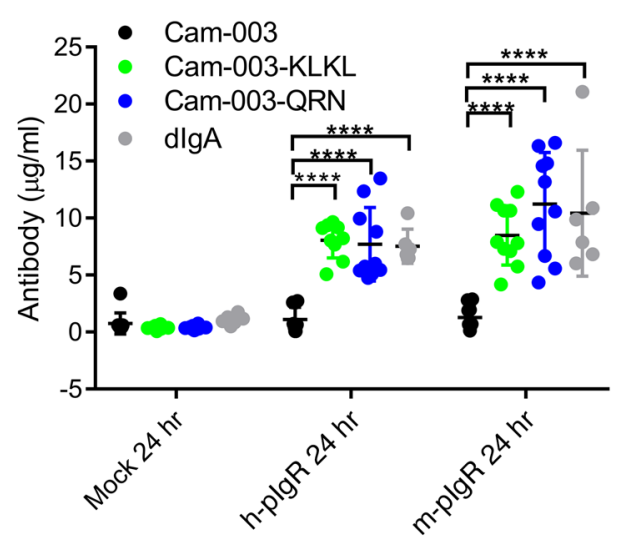

Figure 2. Transcytosis of Cam-003 peptide fusions in plgR-transfected MDCK cells. Antibodies were added to the bottom chamber (basolateral) of transwells containing either untransfected, h-plgR-, or m-plgR-transfected Madin-Darby canine kidney (MDCK) monolayers (A). At 24 hours, antibodies in the top chamber (apical) were quantified by ELISA. Cam-003 levels were similar in all 3 cell lines, whereas Cam-003-QRN and Cam-003-KLKL, as well as human dimeric IgA, had increased accumulation in the apical chamber (B). Data are representative of at least 2 independent experiments $(n=10$ for Cam003 antibodies, $n=6$ for dlgA). Significance was determined using 2-way ANOVA (with Tukey's post hoc test; ${ }^{* * *} P<0.0001$ ).

as well as Cam-003, were then tested for their ability to protect against lethal $P$. aeruginosa infection in a murine acute pneumonia model following prophylactic administration of the antibodies. A highly pathogenic strain of $P$. aeruginosa (strain 6077) was delivered i.n. 24 hours after i.v. administration of either Cam-003, Cam-003-QRN, or the isotype control antibody R347, and survival was monitored. In this initial experiment, both Cam-003-QRN and the parental Cam-003 mAbs at both concentrations exhibited significant enhanced survival when compared with the control IgG (Figure 4B). Moreover, Cam-003-QRN at both 10 and $5 \mathrm{mg} / \mathrm{kg}$ yielded enhanced protective activity when compared with the parental Cam-003 $\mathrm{mAb}$ at each antibody concentration (Figure 4B). We next sought to evaluate the activity of Cam-003-KLKL using a similar experimental design. In this experiment, both Cam-003-KLK-dosed groups ( 6 and $2 \mathrm{mg} / \mathrm{kg})$ survived challenge (Figure 4C). This $100 \%$ protective effect for Cam-003-KLKL was a statistically significant when compared with both the isotype control and the parental Cam-003 molecule at both the 2 and $6 \mathrm{mg} /$ $\mathrm{kg}$ dosage levels. A higher infectious inoculum, approximately 4 -fold above the $100 \%$ lethal dose $\left(\mathrm{LD}_{100}\right)$ for this strain $\left(8 \times 10^{5} \mathrm{CFU} /\right.$ mouse $)$, was then administered to assess whether the survival improvement over the parental Cam-003 afforded by Cam-003-KLKL would persist with greater lung burden. Cam-003 again had a significant protective effect compared with the isotype control at both dosage levels; however, all Cam-003-treated animals succumbed to infection within 50 hours (Figure 4D). Cam-003-KLKL-dosed animals again showed improved survival compared with both the isotype control and the parental molecule Cam-003. Even with an infectious inoculum at 4 times the $\mathrm{LD}_{100}$ for strain 6077 , multiple animals within the Cam-003-KLKL groups survived the challenge until the end of the study, again demonstrating superiority to the parental molecule.

\section{Discussion}

The pIgR plays a key role in mucosal immunity by transporting large amounts of oligomeric $\operatorname{IgA}$ and $\operatorname{IgM}$ into the lumen of organs such as lungs and intestine. In addition to these endogenous pIgR ligands, IgG antibodies (23), Fabs (24-26), single-chain variable fragments (scFvs) (27, 28), camelid VHH domains (29), and phage-derived peptides $(20,21)$ have been generated over the last 2 decades by several laboratories to 


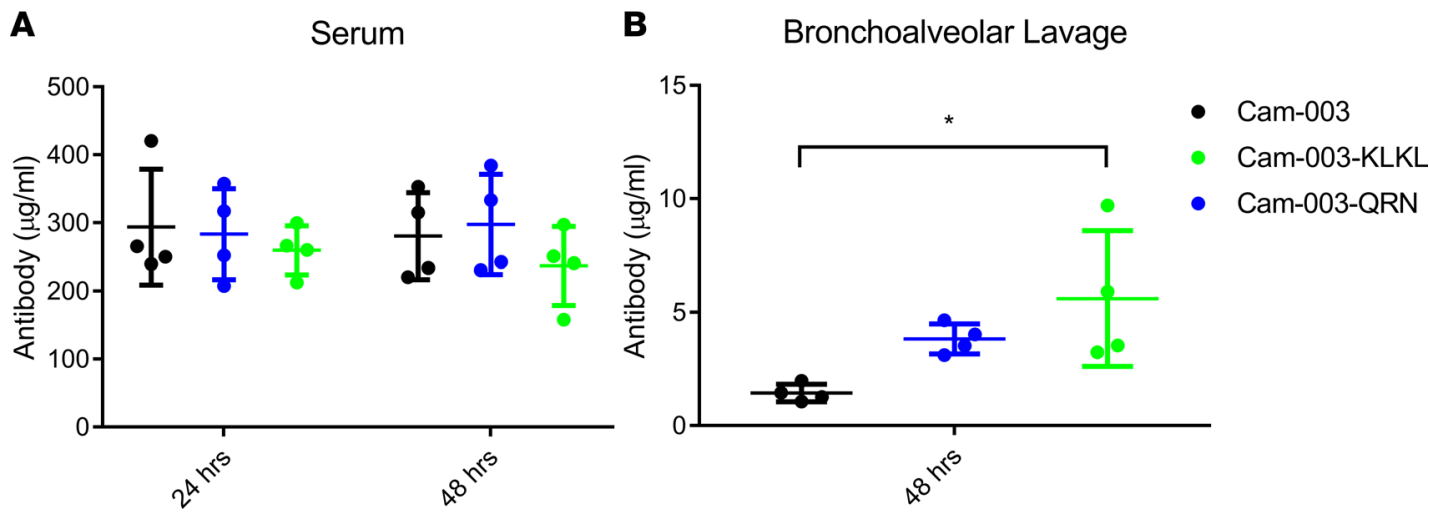

Figure 3. Increased levels of Cam-003 variants in bronchoalveolar lavage and similar levels in serum. (A) Serum levels of Cam-003, Cam-003-QRN, and Cam-003-KLKL were quantified from BALB/c mice 24 and 48 hours after initial i.v. injection. Serum levels did not differ significantly at 24 or 48 hours. (B) Cam-003-QRN and Cam-003-KLKL levels in BAL (assessed at 48 hours) were elevated from those of Cam-003 alone $(P=0.195$ and 0.023 , respectively; 1-way ANOVA with Tukey's post hoc test; $\left.{ }^{*} P<0.05\right)$. Data are representative of at least 2 independent experiments; $n=4$ per group.

bind and become cargo for pIgR-mediated transport. Most of these engineered pIgR ligands show some level of transcytosis in cell monolayers, but few of these have been tested in vivo as to whether they can be transported to mucosal organs. In a notable exception, Fab domains targeting pIgR conjugated to polylysine and bound to condensed plasmid DNA were used to for gene delivery to the mouse lung lumen after i.v. administration (25). In that study, protein expression of the delivered gene was highly elevated in the lung lumen compared with the same complex with a nontargeting Fab. This unique proof-of-concept work showed for the first time to our knowledge that pIgR targeting can lead to accumulation of cargo in the lung lumen upon systemic administration.

Our work herein expands on these results by using a human IgG antibody, Cam-003, as cargo to be transported to the lung lumen by high-affinity pIgR-binding peptides. Cam-003 targets the Psl exopolysaccharide of $P$. aeruginosa and has been shown to mediate protection in a murine model of acute pneumonia (15). We show that, by appending pIgR binding capabilities to Cam-003, we increase its levels in BAL fluid and that this enhanced accumulation correlates with improved protective activity when compared with the parental IgG. This result underscores the potential for this approach to improve the efficacy of antibodies with targets in the lung lumen.

In this study, we screened multiple peptides reported to bind and be transported by $\mathrm{pIgR}$ for their ability to be expressed as IgG fusions and maintain pIgR binding. Only the QRN peptide met these criteria. The KLKL peptide offered a modest improvement in full-length peptide content compared with QRN. Ultimately, further peptide engineering will be needed to both eliminate protease susceptibility and identify optimal attachment sites to IgG for mitigating any potential decreases in Fc $\gamma R$ or $\mathrm{Clq}$ binding. The binding of peptide-IgG fusions to Fc $\gamma \mathrm{R}$ or $\mathrm{C} 1 \mathrm{q}$ was not assessed because OPK activity was minimally affected by the addition of peptides; however, the potential exists that effector function could be impaired by attaching peptides or other fusions to the Fc. As an alternative to peptide engineering, we predict a similar result could be achieved with bispecific antibodies (with 1 or more pIgR-targeting arms) rather than peptides.

A dIgA format (a natural pIgR ligand) has recently been used to deliver antibody to mouse kidney cysts, tissue that is relatively inaccessible to IgG antibodies (30). We predict a similar result could be achieved using our approach to endowing existing IgG antibodies with the ability to bind and be transported by pIgR. An IgG-based approach could be advantageous compared with dIgA due to IgG's favorable pharmacokinetics, relative ease of manufacture, and homogeneity. Indeed, serum levels of Cam-003QRN and Cam-003-KLKL at 24 and 48 hours were similar to those of the parental IgG. dIgA is known to have a much quicker serum clearance ( 4-5 times faster) (31). As our study and another (25) have shown, systemic administration of pIgR-targeting antibodies or fragments increases accumulation in lungs. In this study, we did not assess accumulation in other mucosal organs such as the kidneys or intestines. Results with dIgA accumulation in kidney cysts (30) confirm pIgR-binding Ig can indeed accumulate in organs other than the lungs. Further studies will be necessary to determine where pIgR-binding Ig accumulate 
A Opsonophagocytic Killing

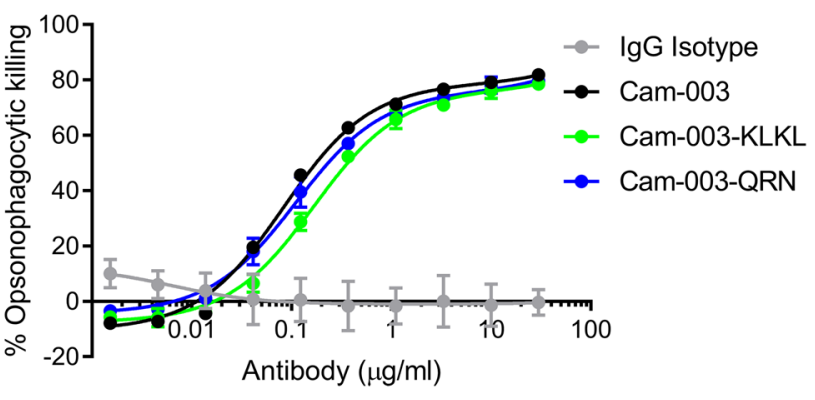

C

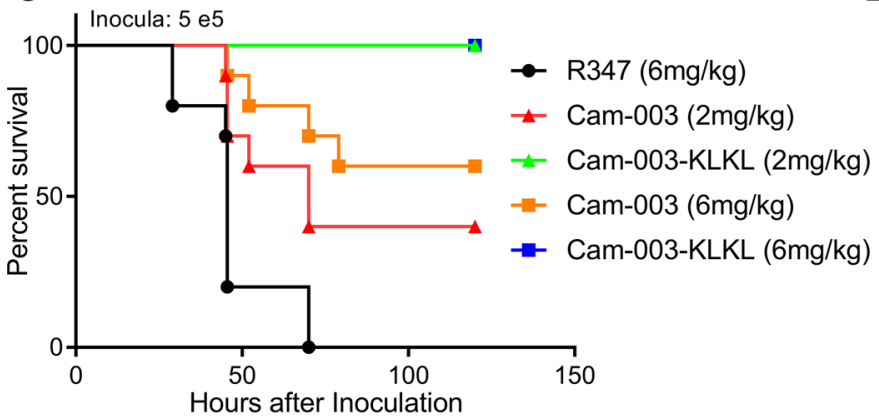

B

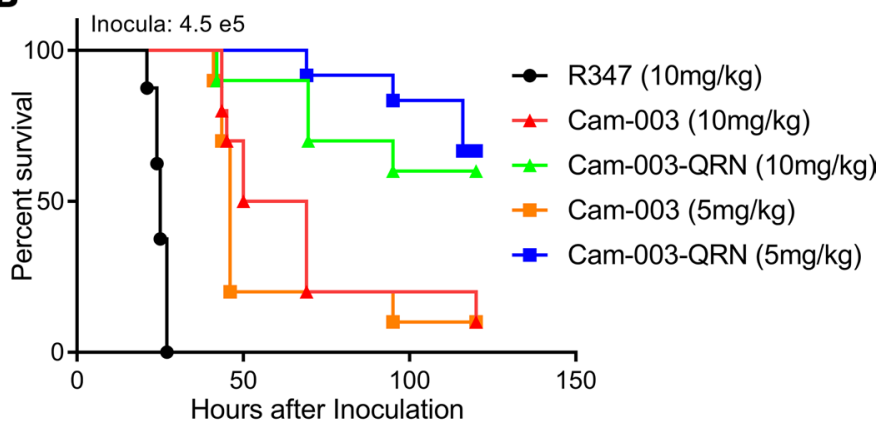

D

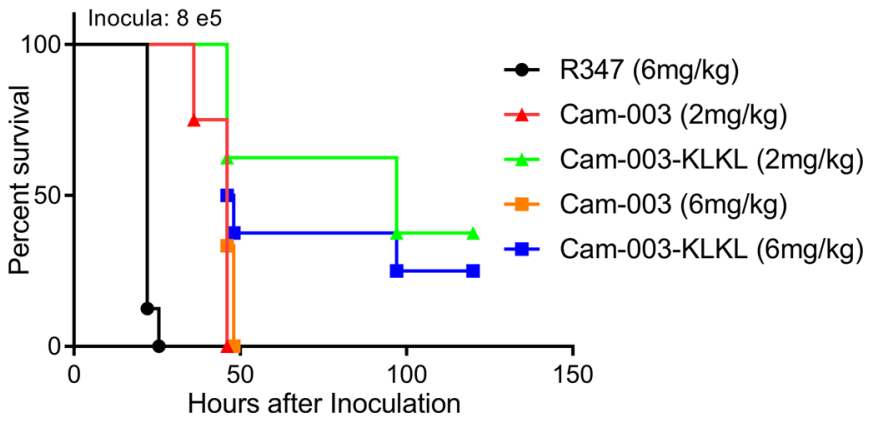

Figure 4. plgR-binding Cam-003 fusions elicit opsonophagocytic killing and improved protection against lethal challenge in a $P$. aeruginosa acute pneumonia model. (A) Opsonophagocytosis assay with luminescent $P$. aeruginosa serogroup 05 strain PA01 (PA01.lux). 0PK with Cam-003-QRN, Cam-003-KLKL, and Cam-003 are shown with the isotype control IgG R347. (B-D) Survival of BALB/c mice prophylactically immunized with an IgG isotype control R347, Cam-003, and either Cam-003-QRN (B) or Cam-003-KLKL (C and D) prior to i.n. infection with P. aeruginosa strain 6077. Results are represented as Kaplan-Meier survival curves; differences in survival were calculated by the log-rank test (Mantel-Cox). Data are representative of at least 2 independent experiments; $n=10$ per group for survival experiments. (B) Cam-003 and Cam-003-QRN had significantly better protection than the isotype control at both doses tested $\left(P<0.0001\right.$ for both 5 and $10 \mathrm{mg} / \mathrm{kg}$ doses; $4.5 \times 10^{5}$ CFU inculcation). Cam-003-QRN survival was significantly improved compared with Cam-003, as well $\left(P=0.0014\right.$ for $5 \mathrm{mg} / \mathrm{kg}$ doses and $P=0.0098$ for $10 \mathrm{mg} / \mathrm{kg}$ doses). (C) At $5 \times 10^{5} \mathrm{CFU}$, Both Cam-003 and Cam-003-KLKL were protective compared with R347 at both doses $(P=0.017,2 \mathrm{mg} / \mathrm{kg}$ Cam-003; $P=0.0004,6 \mathrm{mg} / \mathrm{kg}$ Cam-003; $P<0.0001,2$ and $6 \mathrm{mg} / \mathrm{kg}$ doses of Cam-003-KLKL). Cam-003-KLKL survival was superior to Cam-003 when comparing both the $2 \mathrm{mg} / \mathrm{kg}$ doses $(P$ $=0.0042)$ and $6 \mathrm{mg} / \mathrm{kg}$ doses $(P=0.017)$. (D) At an $8 \times 10^{5} \mathrm{CFU}$ inoculum dose, Cam-003 and Cam-003-KLKL survival was again enhanced compared with R347 ( $P<0.0001,2 \mathrm{mg} / \mathrm{kg}$ Cam-003; $P=0.0002,6 \mathrm{mg} / \mathrm{kg}$ Cam-003; $P<0.0001$, Cam-003-KLKL), with Cam-003-KLKL survival also enhanced compared with Cam-003 ( $P=0.017,6 \mathrm{mg} / \mathrm{kg}$; not significant, $2 \mathrm{mg} / \mathrm{kg})$.

upon various routes of administration (ROA) and whether a specific ROA can cause more accumulation in specific mucosal tissues versus others. Nevertheless, the ability to access mucosal surfaces that have previously been poorly accessible to IgG therapeutics presents an opportunity for enhancing efficacy to both microbial and endogenous targets within these tissues.

\section{Methods}

Cloning. C-terminal peptide fusions were generated using overlapping PCR with synthetic oligonucleotides synthesized at Integrated DNA Technologies. PCR fragments were cloned into a MedImmune proprietary mammalian expression vector (32). Standard molecular biology methods were used for cloning, vectors preparation, and DNA sequencing. E. coli One Shot Stbl3 chemically competent cells, used for DNA amplification, were purchased from Thermo Fisher Scientific.

Expression and purification. Antibodies were expressed as previously described (33). Briefly, expression vectors expressing both antibody light and heavy chains were transfected in CHO-G22 cells. CHO-G22 cells were cultured with $25 \mu \mathrm{M}$ L-methionine sulfoximine and $100 \mu \mathrm{g} / \mathrm{ml}$ hygromycin in MedImmune's proprietary $\mathrm{CHO}-\mathrm{V} 2$ medium at $37^{\circ} \mathrm{C}, 5 \% \mathrm{CO}_{2}$, and $80 \%$ humidity. Twenty-four hours prior to transfection, CHO-G22 cells were diluted to $1 \times 10^{6} \mathrm{cells} / \mathrm{ml}$ in $\mathrm{CHO}-\mathrm{V} 2$ medium without hygromycin and L-methionine sulfoximine and grown overnight. mAb expression vector $(500 \mu \mathrm{g})$ was diluted into 7.5 $\mathrm{ml}$ of $150 \mathrm{mM}$ sodium chloride, and $2.5 \mathrm{ml}$ of $1 \mathrm{mg} / \mathrm{ml}$ PEI MAX (Polysciences) was mixed with 5 $\mathrm{ml}$ of the $150 \mathrm{mM}$ sodium chloride solution (VWR). The DNA and PEI-max solutions were combined and incubated for 1 minute at room temperature before being added to $500 \mathrm{ml}$ of $2 \times 10^{6} \mathrm{CHO}-\mathrm{G} 22$ 
cells. The transfected cells were grown for 24 hours as described above. Thereafter, the temperature was changed to $34^{\circ} \mathrm{C}$ and supplemented with $500 \mathrm{ml}$ of CHO-V2 medium. Culture medium was collected 10 days after transfection. Antibody variants were purified using standard protein A affinity chromatography and were subsequently buffer exchanged using dialysis in PBS ( $\mathrm{pH}$ 7.2). Monomeric content of the antibodies and antibody fusions were analyzed using analytical size-exclusion chromatography (SEC-HPLC). Antibodies having an initial monomer content below 98\% had aggregate removed by preparative SEC-HPLC prior to testing (Supplemental Figure 2).

ELISA binding to pIgR. Human or murine pIgR extracellular domain (R\&D Systems) was coated on 96-well maxisorp plates (Nunc) overnight at $4^{\circ} \mathrm{C}(2 \mu \mathrm{g} / \mathrm{ml}$ in PBS). Plates were then blocked with $2 \%$ BSA in PBS for 2 hours at room temperature. After washing, titrated antibodies or antibody fusions were added and allowed to incubate for 1 hour. After 3 washes in PBS, peroxidase-conjugated anti-human Fd (Southern Biotech, catalog 2046-05) was used to as a detection reagent. The wells were washed 3 times, and TMB substrate solution was added. The reaction was stopped with $2 \mathrm{~N} \mathrm{H}_{2} \mathrm{SO}_{4}$, and the absorbance at $450 \mathrm{~nm}$ was measured.

Transcytosis assay. MDCK (purchased from ATCC) transcytosis experiments were conducted similarly to Jerdeva et. al (34). Stably transfected MDCK cells expressing either murine or human full-length pIgR were plated in transwell plates with at 500,000 cells/well with MEM $+10 \%$ or FBS $+1 \%$ penicillin-streptomycin. MDCK pIgR cells were generated in house using a lentiviral vector and confirmed to have pIgR expression. Media ( $1 \mathrm{ml}$ ) was added on the top (apical) side; $0.5 \mathrm{ml}$ of media was added on the bottom (basolateral) side. Media was changed every 48 hours prior to beginning transcytosis experiment. Cells were used for experiments on the fourth or fifth day after plating, after confluent monolayers had formed. Antibody $(20 \mu \mathrm{g})$ was added to the bottom chamber, and media was collected from the top chamber; antibody amount was assessed by OCTET or quantitative ELISA.

$B A L$ and serum collection and quantification. BALB/c mice (6-8 weeks old; Envigo) were given a bolus i.v. dose of $2.5 \mathrm{mg} / \mathrm{kg}$ antibody. Four mice were used per antibody. Blood samples were obtained from the retro-orbital plexus using capillary pipettes at 24 hours and via cardiac puncture at 48 hours. BAL was taken at 48 hours by washing the lung lumen with $1 \mathrm{ml}$ of PBS $3 \times$ and collecting. Biolayer interferometry (OCTET; FortéBio Inc.) was used to monitor the serum or BAL concentrations of the tested antibodies. Briefly, samples or buffer were dispensed into 384-well microtiter plates (FortéBio Inc.) at a volume of $50 \mu \mathrm{l}$ per well. Operating temperature was maintained at $30^{\circ} \mathrm{C}$. Anti-human Fab-coated biosensor tips (FortéBio Inc.) were prewetted with PBS. Standard curves were generated for each antibody variant, generated via OCTET analysis software. Sample concentrations were generated automatically based on the standard curve via the OCTET User Software (version 3.1) and was subsequently analyzed via Prism (GraphPad Software).

OPK assay. Assays were performed as previously described, with some modifications (15). OPK assays were performed in a 96-well plate format. P. aeruginosa strain PA01-lux (expressing luciferase) was constructed as previously reported (35) and grown to mid log-phase in LB media, and cultures were diluted to $2 \times 10^{6}$ cells $/ \mathrm{ml}$. Bacteria $(25 \mu \mathrm{l})$ were then combined with an equal volume of $10 \%$ baby rabbit serum; differentiated HL-60 cells $\left(2 \times 10^{7}\right.$ cells $\left./ \mathrm{ml}\right)$ as the polymorphonuclear leukocyte source; and titrated $\mathrm{mAb}$. After 2-hour shaking at $37^{\circ} \mathrm{C}$, relative luciferase units (RLUs) for each well were determined using an Envision Multilabel plate reader (PerkinElmer). The percentage of killing was determined by comparing RLUs derived from assays lacking mAb (no OPK) to RLUs obtained from assays with anti-Psl mAbs or the control R347 mAb (generated in-house). For OPK assays using human sera as a complement source, human serum was first adsorbed against an isogenic Psl-deficient mutant strain (PAO1 $\Delta$ pslA) (15) and filter sterilized $(0.2 \mu \mathrm{m})$. Adsorbed sera was then used in place of rabbit sera in the OPK assay described above. HL-60 cells were purchased from ATCC.

In vivo $P$. aeruginosa model. Studies were performed essentially as described previously $(15,36)$ with some modifications. Guidelines for humane end points were strictly followed. Antibodies were administered 24 hours before infection with $P$. aeruginosa strain 6077 in the acute pneumonia model. BALB/c mice (Envigo) were infected with 6077 suspended in a 0.05-ml inoculum. Strain 6077 was a gift from Joanna Goldberg (Emory University, Atlanta, Georgia, USA).

Statistics. Data were analyzed using 1-way ANOVA with Tukey's post hoc test or 2-way ANOVA with Tukey's post hoc test. For survival studies, data are presented as Kaplan-Meier survival curves and were analyzed by the log-rank test. All statistical analyses were performed using GraphPad Prism version 7 software. 
Study approval. All animal studies were approved by the MedImmune IACUC and were conducted in an Association for Assessment and Accreditation of Laboratory Animal Care-accredited facility in compliance with US regulations governing the housing and use of animals. Donor serum was obtained using a MedImmune approved IRB protocol (IRB Pro00004341).

\section{Author contributions}

$\mathrm{MJB}, \mathrm{AD}$, and RG designed experiments, performed experiments, analyzed data, and wrote the manuscript. GMM and NB performed experiments. MPM designed experiments. NB, MJB, KJL, and SSP produced the antibodies or cell lines. ASB performed mass spectrophotometric analysis. PT, HW, and WFD wrote the manuscript.

\section{Acknowledgments}

Research was supported by MedImmune, a member of the AstraZeneca group.

Address correspondence to: M. Jack Borrok or Ping Tsui, One MedImmune Way, Gaithersburg, Maryland 20878, USA. Phone: 301.398.5460; Email: borrokm@medimmune.com (MJB). Phone: 301.398.5245; Email: ping.tsui.eng@gmail.com (PT).

1. Shadman KA, Wald ER. A review of palivizumab and emerging therapies for respiratory syncytial virus. Expert Opin Biol Ther. 2011;11(11):1455-1467.

2. DiGiandomenico A, et al. A multifunctional bispecific antibody protects against Pseudomonas aeruginosa. Sci Transl Med. 2014;6(262):262ra155

3. Hua L, et al. MEDI4893* Promotes Survival and Extends the Antibiotic Treatment Window in a Staphylococcus aureus Immunocompromised Pneumonia Model. Antimicrob Agents Chemother. 2015;59(8):4526-4532.

4. Antoniu SA. Monoclonal antibodies for asthma and chronic obstructive pulmonary disease. Expert Opin Biol Ther. 2013;13(2):257-268

5. Denmark VK, Mayer L. Current status of monoclonal antibody therapy for the treatment of inflammatory bowel disease: an update. Expert Rev Clin Immunol. 2013;9(1):77-92.

6. Hart TK, et al. Preclinical efficacy and safety of mepolizumab (SB-240563), a humanized monoclonal antibody to IL-5, in cynomolgus monkeys. J Allergy Clin Immunol. 2001;108(2):250-257.

7. Peebles RS, Liu MC, Lichtenstein LM, Hamilton RG. IgA, IgG and IgM quantification in bronchoalveolar lavage fluids from allergic rhinitics, allergic asthmatics, and normal subjects by monoclonal antibody-based immunoenzymetric assays. J Immunol Methods. 1995;179(1):77-86.

8. Wu H, et al. Development of motavizumab, an ultra-potent antibody for the prevention of respiratory syncytial virus infection in the upper and lower respiratory tract. J Mol Biol. 2007;368(3):652-665.

9. Monteiro RC, Van De Winkel JG. IgA Fc receptors. Annu Rev Immunol. 2003;21:177-204.

10. Norderhaug IN, Johansen FE, Schjerven H, Brandtzaeg P. Regulation of the formation and external transport of secretory immunoglobulins. Crit Rev Immunol. 1999;19(5-6):481-508.

11. Woof JM, Russell MW. Structure and function relationships in IgA. Mucosal Immunol. 2011;4(6):590-597.

12. Crottet $P$, Corthésy B. Secretory component delays the conversion of secretory IgA into antigen-binding competent $F(a b ') 2$ : a possible implication for mucosal defense. J Immunol. 1998;161(10):5445-5453.

13. Phalipon A, Cardona A, Kraehenbuhl JP, Edelman L, Sansonetti PJ, Corthésy B. Secretory component: a new role in secretory IgA-mediated immune exclusion in vivo. Immunity. 2002;17(1):107-115.

14. Underdown BJ, Dorrington KJ. Studies on the structural and conformational basis for the relative resistance of serum and secretory immunoglobulin A to proteolysis. J Immunol. 1974;112(3):949-959.

15. DiGiandomenico A, et al. Identification of broadly protective human antibodies to Pseudomonas aeruginosa exopolysaccharide Psl by phenotypic screening. J Exp Med. 2012;209(7):1273-1287.

16. Byrd MS, et al. Genetic and biochemical analyses of the Pseudomonas aeruginosa Psl exopolysaccharide reveal overlapping roles for polysaccharide synthesis enzymes in Psl and LPS production. Mol Microbiol. 2009;73(4):622-638.

17. Matsukawa M, Greenberg EP. Putative exopolysaccharide synthesis genes influence Pseudomonas aeruginosa biofilm development. J Bacteriol. 2004;186(14):4449-4456.

18. Mishra M, et al. Pseudomonas aeruginosa Psl polysaccharide reduces neutrophil phagocytosis and the oxidative response by limiting complement-mediated opsonization. Cell Microbiol. 2012;14(1):95-106.

19. Thanabalasuriar A, et al. Bispecific antibody targets multiple Pseudomonas aeruginosa evasion mechanisms in the lung vasculature. J Clin Invest. 2017;127(6):2249-2261.

20. Braathen $\mathrm{R}$, et al. Identification of a polymeric Ig receptor binding phage-displayed peptide that exploits epithelial transcytosis without dimeric IgA competition. J Biol Chem. 2006;281(11):7075-7081.

21. White KD, Capra JD. Targeting mucosal sites by polymeric immunoglobulin receptor-directed peptides. J Exp Med. 2002;196(4):551-555.

22. Oztan A, Rondanino C, Apodaca G. Transcytosis of polymeric immunoglobulin a in polarized Madin-Darby canine kidney cells. Methods Mol Biol. 2008;440:157-170.

23. Gupta S, Heacock M, Perez A, Davis PB. Antibodies to the polymeric immunoglobulin receptor with different binding and 
trafficking patterns. Am J Respir Cell Mol Biol. 2005;33(4):363-370.

24. Ferkol T, Kaetzel CS, Davis PB. Gene transfer into respiratory epithelial cells by targeting the polymeric immunoglobulin receptor. J Clin Invest. 1993;92(5):2394-2400.

25. Ferkol T, Perales JC, Eckman E, Kaetzel CS, Hanson RW, Davis PB. Gene transfer into the airway epithelium of animals by targeting the polymeric immunoglobulin receptor. J Clin Invest. 1995;95(2):493-502.

26. Song W, Bomsel M, Casanova J, Vaerman JP, Mostov K. Stimulation of transcytosis of the polymeric immunoglobulin receptor by dimeric IgA. Proc Natl Acad Sci USA. 1994;91(1):163-166.

27. Ferkol T, Cohn LA, Phillips TE, Smith A, Davis PB. Targeted delivery of antiprotease to the epithelial surface of human tracheal xenografts. Am J Respir Crit Care Med. 2003;167(10):1374-1379.

28. Ferkol T, Eckman E, Swaidani S, Silski C, Davis P. Transport of bifunctional proteins across respiratory epithelial cells via the polymeric immunoglobulin receptor. Am J Respir Crit Care Med. 2000;161(3 Pt 1):944-951.

29. Emmerson CD, et al. Enhancement of polymeric immunoglobulin receptor transcytosis by biparatopic VHH. PLoS ONE. 2011;6(10):e26299.

30. Olsan EE, Matsushita T, Rezaei M, Weimbs T. Exploitation of the Polymeric Immunoglobulin Receptor for Antibody Targeting to Renal Cyst Lumens in Polycystic Kidney Disease. J Biol Chem. 2015;290(25):15679-15686.

31. Fouda GG, et al. Systemic administration of an HIV-1 broadly neutralizing dimeric IgA yields mucosal secretory IgA and virus neutralization. Mucosal Immunol. 2017;10(1):228-237.

32. Thompson $P$, et al. Rational design, biophysical and biological characterization of site-specific antibody-tubulysin conjugates with improved stability, efficacy and pharmacokinetics. J Control Release. 2016;236:100-116.

33. Dimasi N, et al. Efficient Preparation of Site-Specific Antibody-Drug Conjugates Using Cysteine Insertion. Mol Pharm. 2017;14(5):1501-1516

34. Jerdeva GV, Tesar DB, Huey-Tubman KE, Ladinsky MS, Fraser SE, Bjorkman PJ. Comparison of FcRn- and pIgR-mediated transport in MDCK cells by fluorescence confocal microscopy. Traffic. 2010;11(9):1205-1220.

35. Choi KH, et al. A Tn7-based broad-range bacterial cloning and expression system. Nat Methods. 2005;2(6):443-448.

36. DiGiandomenico A, et al. Intranasal immunization with heterologously expressed polysaccharide protects against multiple Pseudomonas aeruginosa infections. Proc Natl Acad Sci USA. 2007;104(11):4624-4629. 